\title{
Estrategias preventivas en relación a las conductas adictivas en adolescentes
}

\author{
Preventive strategies for addictive behaviors in teenagers
}

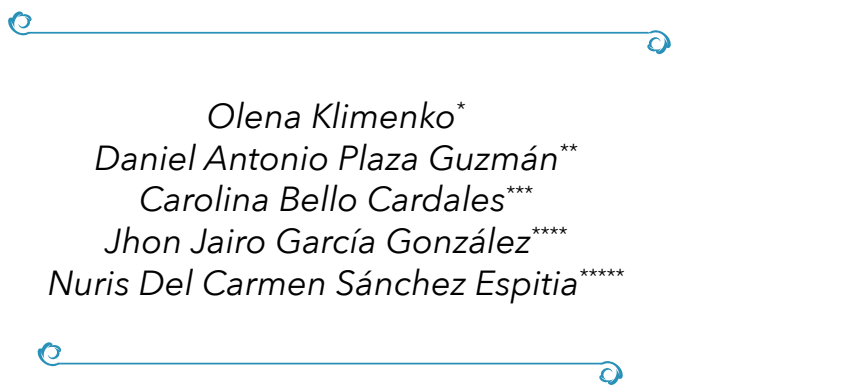

Recibido: $12.05 .2018 \bullet$ Arbitrado: $10.06 .2018 \bullet$

Aprobado: 15.06 .2018

Psicóloga, Magister en Ciencias Sociales, Doctoranda en Psicopedagogía, Docente Institución Universitaria de Envigado, Docente UCLA, olenak45@ gmail.com, ORCID: 0000-0002-84111263

* Psicólogo, Especialista en Adicciones, danieladicciones2017@gmail.com

*** Psicóloga, Especialista en Adicciones, caro_20010@hotmail.com

**** Psicólogo, Especialista en Adicciones, jhongar27@hotmail.com

${ }^{* * * * *}$ Psicóloga, Especialista en Adicciones, nuris.psicologia@gmail.com

\section{Resumen}

Artículo presenta una revisión bibliográfica acerca de las estrategias preventivas en relación a las conductas adictivas en adolescentes, a partir de una metodología e enfoque cualitativo y método documental. Se realizó una revisión sistemática de documentos en español e inglés, relacionados con el objeto de estudio, en las bases de Scopus, Ebsco, Science Direct, Scielo, NDLTD, entre otros, mediante la técnica del "rastreo secuencial". Se pudo concluir que la prevención temprana desde el área personal, familiar o comunitaria se presenta como alternativa viable para mantener alejado al adolescente de adicciones que causan daños irreparables en su comportamiento y convivencia. Así mismo, se le debe seguir apostando a las estrategias preventivas duraderas y estables en el tiempo con el fin generar impacto a largo plazo tanto en relación a adiciones químicas como comportamentales.

Palabras clave: adicción, prevención, adolescentes, estrategias, familia. 


\section{Abstract}

The purpose of this article is to carry out a bibliographic review exposed by various authors about preventive strategies in relation to addictive behaviors in adolescents, based on a study methodology with a qualitative approach and a documentary method. A systematic review of documents in Spanish and English was carried out, related to the object of study, in the bases of Scopus, Ebsco, Science Direct, Scielo, NDLTD, among others, through the technique of "sequential tracking". It was concluded that early prevention from the personal, family or community are presented as viable alternatives to keep the adolescent away from addictions that only cause irreparable damage to their behavior and coexistence. Likewise, is should continue betting to stable and lasting preventive strategies at the time in order to generate long-term impact both in relation to chemical additions such as behavioral.

Keywords: addiction, prevention, adolescents, strategies, family.

\section{Introducción}

El fenómeno de adicción es bastante complejo y no puede explicarse sobre la base de causas únicas, considerándose como un fruto de la interacción conjunta de diferentes tipos de factores: variables o dimensiones de personalidad y variables sociales y contextuales (familiares, escolares y grupales) (López, Vilariño, Linares y González, 2014).

En la última década han sido muchas las líneas de investigación dirigidas al estudio del fenómeno de adición tanto para sustancias, como comportamental, intentando determinar factores que favorecen y/o se relacionan con este (Muñoz y Graña, 2001). Algunos estudios apuntan, por ejemplo, a la relación de adiciones con el funcionamiento ejecutivo (Mariño, Castro y Torrado, 2012); rol de bases neurobiológicas en la adicción (Ayesta, 2002), contribución de la resiliencia a las conductas adictivas (Becoña, 2007); relación entre las creencias asociadas al consumo y dependencia de sustancias (Vargas y Londoño, 2016; Martínez y Verdejo, 2011); influencia de las actitudes favorables hacia el consumo en la generación de comportamiento adictivo (Ortiz, Lopera, González y Klimenko, 2017; Melo y Castanheira, 2010); comorbilidad y patología dual en el consumo (Albein-Uriosa, Martínez-González, Gutiérrez, Lozano y Verdejo-García, 2011), influencia de cultura contemporánea en las adiciones no comportamentales (Jáuregui, 2002; Otero-López y Villardefranco, 2011); particularidades del consumo de drogas en adolescencia y edad joven (Becoña 1999; 2007), entre otros. 
Los autores resaltan múltiples factores que inciden en el inicio y mantenimiento de la conducta adictiva, tanto en relación a las adiciones químicas, como comportamentales, relacionados con diferentes ámbitos de la vida. En cuanto a los factores individuales, se resalta la participación de rasgos de personalidad (Gonzálvez, Espada, Guillén-Riquelme, Secades y Orgilés, 2016); la edad y género, siendo la edad adolescente y el género masculino de mayor riesgo para el inicio del consumo (Vargas, Londoño, 2016). Se considera también la influencia de las actitudes, creencias y valores que se manejan al respecto del consumo de sustancias y/o diferentes comportamientos adictivos (Ortiz et al.,

2017; De la Villa Moral y Ovejero, 2005); percepción de riesgo de consumo (De la Villa Moral, Rodríguez y Ovejero, 2010); manejo de estrategias de afrontamiento ineficaces (Llorens, Perelló y Palmer, 2004); problemas a nivel de autoestima, autoconcepto y autocontrol (López-Torrecillas, Peralta, Muñoz-Rivas y Godoy, 2003), entre otros.

Por otra parte, en el ámbito familiar se nombran algunos factores como: el modo de enfrentar los problemas a nivel familiar, y dentro de estos, la falta de apoyo religioso y de la búsqueda de apoyo profesional, conjuntamente con las dificultades de comunicación intrafamiliar (Cid-Monckton y Pedrao, 2011); las pautas de interacción familiar, como la comunicación deficiente, los frecuentes conflictos, la baja cohesión, el excesivo castigo o el consumo de los propios padres (Musitu, Jiménez y Murgui, 2007). La familia emerge como núcleo desencadenante de conductas de riesgo, si el adolescente la percibe como un ambiente hostil busca un grupo que le brinde seguridad, pero al carecer de una referencia fiable de sus propios atributos, presenta un proceso de desadaptación provocándole el rechazo de otros. Esto deriva en la exposición a factores de riesgo como el consumo de drogas, la promiscuidad y conductas violentas (Gómez Cobos, 2008).

Desde lo social-comunitario también se dirige la atención a la participación de factores como, por ejemplo, los significados que los sujetos otorgan a las prácticas de riesgo y cuidado a partir de su pertenencia a determinados contextos socioculturales (Camarotti y Kornblit, 2015), o la incidencia de ciertos movimientos sociales en la posición respecto del consumo de sustancias psicoactivas (Slapak y Grigoravicius, 2007).

En el ámbito escolar también se puede facilitar y/o prevenir el surgimiento de conductas adictivas. En esta línea, los autores resaltan variados factores que inciden en el consumo de los adolescentes, entre ellos se puede resaltar la desadaptación al contexto escolar, las dificultades en las relaciones 
estudiante-profesor, el fracaso escolar y el acoso en la escuela (Gómez, Gutiérrez y Londoño, 2013).

Además de los aspectos mencionados anteriormente, cuando se trata de acercarse al fenómeno de consumo, es necesario considerar la variable de la edad evolutiva, considerando sus características particulares que pueden contribuir al aumento de factores de riesgo para el consumo. En este caso, la adolescencia es una edad particularmente vulnerable, asociada con el surgimiento de diferentes problemáticas, incluyendo el inicio de conductas adictivas (Alfonso, Huedo-Medina y Espada, 2009). Existen numerosos factores en el universo adolescente que estarían disparando ciertas conductas de riesgo e influenciando, en parte, en algunas de las patologías más frecuentes de esta edad. Estos factores tienen un origen tanto interno como externo, e incluyen aspectos personales, familiares y sociales (Páramo, 2011). Los factores biológicos y sociales o medioambientales interactúan en la adicción a las drogas en la adolescencia (Wille- González, 2011). En cuanto a los factores de riesgos para el consumo de drogas ilegales en la adolescencia, las investigaciones indican que el consumo de sustancias en la adolescencia se relaciona con un déficit en recursos personales, con una mala calidad del clima, la comunicación y la afectividad en la familia, dificultades de integración social y falta de apoyos (Rueda y Monreal, 2012), al igual como la presión del grupo, disminución en la autoestima (autoconcepto y autoeficacia), desintegración familiar, la creciente necesidad de autonomía, rechazo a la protección adulta, estrés, cambios asociados al desarrollo, entre otros (Musayon, Torres, Sanchez y Chavez, 2005).

En el caso de la adolescencia, el consumo abusivo de alcohol supone un problema de salud pública con características específicas que requieren medidas preventivas, debido a las formas que adopta este consumo en muchos grupos de jóvenes (Villarreal, Sánchez y Musitu, 2013). La adolescencia representa una etapa crítica en el inicio a la experimentación de drogas iniciáticas (alcohol y tabaco) y, en menor medida, de otras sustancias (Jiménez y Ovejero, 2003). La adolescencia es descrita por algunos autores como un período de experimentación y, como tal, es un momento en que los adolescentes exploran distintas alternativas (de ocio, de relaciones sociales y amorosas, etc.) entre las que se encuentran las conductas de riesgo (Villarreal et al., 2013). Se considera que la tendencia a la búsqueda de sensaciones representa uno de los principales motivadores de consumo, tanto de alcohol como de otras drogas, de modo que la curiosidad por la experimentación y la conducta impulsiva constituyen factores que determinan la iniciación al consumo (De la Villa Moral, Rodríguez y Ovejero, 2010). 
Considerando la complejidad del fenómeno de consumo, y más aún, el consumo en la edad de adolescencia, el acercamiento al tema de estrategias preventivas debe considerar múltiples factores intervinientes y agravantes del fenómeno, con el fin de que estas estrategias sean realmente efectivas y funcionales.

Por tanto, los programas preventivos son un conjunto de actuaciones con el objetivo específico de impedir la aparición del problema al que se dirige dicho programa preventivo, lo cual, en el caso de las drogodependencias, cosiste en impedir o retrasar la aparición de conductas de consumo, uso y abuso de distintas drogas. Un programa preventivo puede ser global para toda la comunidad o específico para un subgrupo de personas, barrio concreto, grupo de edades, etc. (Becoña, 1999).

Algunos autores indican la baja eficencia de programas de prevención existentes, afirmando que las características principales y la ejecución de las políticas públicas que se han empleado como estrategias de prevención para el consumo de sustancias psicoactivas, no han tenido un gran impacto ni generan efectividad en la población a la cual se orientan, permitiendo, incluso en algunos casos, la ampliación de consumo, discutiendo los efectos iatrogénicos de los programas de prevención de alcohol y drogas (Arco y Fernández, 2002).

Cabe resaltar que las cifras presentadas en el informe de la Oficina de Naciones Unidas contra la Droga y el Delito (UNODC, 2015), refuerzan la idea anterior. Por primera vez en seis años, la cifra de personas con problemas graves con las drogas aumentó "de forma desproporcionada" hasta los 29,6 millones de personas, frente a los 27 millones de

2013. Además, el documento indica que en el mundo hay 307 millones de consumidores de algún tipo de droga. Estos números parecen confirmar que la llamada guerra contra las drogas liderada por Estados Unidos fracasó desde el punto de vista humano.

Por otra parte, diferentes estudios realizados en Colombia, muestran un incremento constante en el consumo de drogas y con edades de inicio cada vez más tempranas (Cáceres, Salazar, Varela y Tovar, 2006). Estudios nacionales reflejan que el consumo de drogas en Colombia a 2013 se sobrepaso a 2,6 millones de personas, lo cual representa a un $11,1 \%$ de la población total (12-65 años), con consumo problemático de alcohol, entre los cuales, 276.367 personas presentan comportamientos indicativos de dependencia; el mayor compromiso se encuentra en el grupo de edad entre los 18 y 24 años, 
indicando que el consumo afecta especialmente jóvenes y adultos en edad productiva (ODC, 2014).

Por otra parte, el Observatorio de Drogas del Ministerio de Justicia (2017) alertó que en el país aumentó el consumo de drogas ilícitas, "no solo porque más personas las consumen sino porque el mercado de sustancias es cada vez más amplio y diverso" (p. 387). El documento "Reporte de Drogas de Colombia 2016" expuso el aumento significativo en el uso de cualquier sustancia ilícita (marihuana, cocaína, bazuco, éxtasis o heroína), tanto en la prevalencia de uso alguna vez en la vida (de $8,8 \%$ en el 2008 a $12,2 \%$ en el 2013), como en uso durante el último año (de $2,6 \%$ a $3,6 \%$ ), indicando un aumento relevante en cuanto a la adicción de sustancia psicoactivas en la población colombiana.

Actualmente el interés de los profesionales de salud mental se dirige también a las adiciones no relacionadas a sustancias, denominadas también como adiciones comportamentales.

Este tipo de adicción consiste en una pérdida de control de la persona ante cierto tipo de conductas que tienen como características producir dependencia, síndrome de abstinencia, tolerancia, vivir para y en función de esa conducta, etc. Este tipo de comportamientos cumplen los criterios clínicos y requieren de tratamiento por la incapacidad que producen en su vida cotidiana, como puede ejemplificarse con la adicción al juego, al comer compulsivo, al sexo y al amor romántico, al trabajo, a las compras, a la televisión, a los videojuegos, al ordenador, a internet, a los teléfonos móviles, a la religión y sectas, etc. (Horcajadas, Castillo y Mangado, 2010; Becoña, 2006; García del Catillo, 2013). Por ejemplo, existe preocupación sobre cada vez creciente afectación de los adolescentes por la adición a nuevas tecnologías (Chóliz Montañés, 2017).

El interés que orienta esta revisión es dar a conocer elementos relevantes de las propuestas de prevención tanto en relación al consumo de sustancias, como en relación a las adicciones no comportamentales, orientadas a la edad adolescente.

\section{Metodología}

El proceso de revisión bibliográfica se clasifica como estudio de enfoque cualitativo y método documental. Se realizó una revisión sistemática de documentos en español e inglés, relacionados con el objeto de estudio, en las bases de Scopus, Ebsco, Science Direct, Scielo, NDLTD, entre otros, mediante la técnica del "rastreo secuencial"(Vara Horna, 2010), con el fin 
de obtener el espacio literario relevante (ELR) (Perez Rave, 2012). Se empleó el análisis intra e intertextual y la ficha bibliográfica como instrumento. Se tuvieron en cuenta normas éticas relacionadas con los derechos de autor y normas de citación de fuentes bibliográficas.

\section{Desarrollo del tema}

En la actualidad la preocupación por disminuir el consumo de sustancias legales e ilegales se ha convertido en un tema de salud pública; siguiendo el informe mundial de la Oficina de Naciones Unidas contra la Droga y el Delito (UNODC, 2015) el cual explica que aproximadamente 27 millones de personas del mundo son drogodependientes, razón por la cual debe considerarse el abuso de estas drogas como una situación social que requiere prevención, tratamiento y atención continua, con el fin de reducir daños y promover el bienestar del ser humano.

Según la Agencia Nacional de Defensa Jurídica Del Estado (2016), la prevención en lo que hace referencia al consumo de Alcohol y Drogas es compromiso de todos; este compromiso incluye la responsabilidad de vigilar y propender por el mutuo control entre todas las personas que pueden estar involucradas en escenarios vulnerables a esta problemática.

En base a esto, las estrategias de prevención buscan generar factores protectores que disminuyan la vulnerabilidad al consumo de alcohol o sustancias psicoactivas (SPA) y las conductas de riesgo asociadas a estos, mediante el avance de habilidades y capacidades para la toma de decisiones, la promoción de hábitos y estilos de vida saludables, así como el fortalecimiento de las redes familiares y comunitarias, la integración y la inclusión social. Por medio de esto se pueden prevenir posibles efectos negativos en individuos, comunidades y la sociedad en general (Llano Sierra et al., 2017).

Cualquier estrategia debe conllevar a planear acciones para reducir factores de riesgo y daños que se presentan en diferentes niveles. Sin embargo, es de resaltar que estos riesgos y daños no precisamente se presentan cuando hay consumo de SPA, siendo una aproximación a las situaciones potenciales a las que se puede enfrentar el individuo consumidor de SPA y su impacto en diferentes niveles. La prevención busca gestionar de la manera más efectiva estos riesgos para evitar daños y consecuencias negativas. En el marco de la protección social, la prevención es una estrategia para manejar o gestionar más efectivamente el riesgo social. Se pone en marcha antes de que el riesgo se manifieste y buscan reducir la probabilidad de que un choque adverso afecte la salud, la calidad de vida y el bienestar de las personas, los hogares 
y las comunidades. En este caso, los "choques" hacen referencia a los factores de riesgo que operan en los niveles macrosociales, microsociales y personales (UNODC, 2010).

\section{Estrategias de prevención a nivel individual}

\section{Formación en habilidades para la vida}

Las lecciones aprendidas de la investigación en adicciones, han reconocido a la prevención universal como la mejor alternativa para combatir el consumo de drogas en el mundo (National Institute of Drug Abuse (NIDA), 2003). Por ello desde hace más de medio siglo, se han desarrollado programas preventivos de consumo de drogas. Actualmente existe una amplia evidencia científica sobre el éxito de los programas escolarizados con un enfoque de habilidades cuyo objetivo ha sido la prevención de consumo de drogas en niños y adolescentes (Botvin y Griffin, 2007).

La Organización Mundial de la Salud (OMS) ha denominado a estas habilidades como "habilidades para la vida", que define como: "aquellas destrezas que permiten que los adolescentes adquieran las aptitudes necesarias para su desarrollo personal y para enfrentar en forma efectiva los retos de la vida diaria" (OMS, 1993) y las ha dividido en tres categorías: sociales, cognitivas y emocionales. Estas habilidades han demostrado ser eficaces no solo en la prevención del consumo de drogas, sino en una amplia gama de conductas de riesgo en niños y adolescentes tales como en la prevención del embarazo adolescente e Infecciones de Transmisión Sexual (ITS), incluyendo al SIDA, prevención de la violencia, entre otras (Mangrulkar, Whitman y Posner 2001; Organización Panamericana de la Salud (OPS), 2001).

Entre los programas desarrollados con un enfoque de habilidades para la vida en el ámbito internacional, destaca el Programa de Entrenamiento en Habilidades para la Vida (Life Skills Training Program, LST). El LST es un programa universal para estudiantes que les enseña habilidades sociales, cognitivas y emocionales, además de proporcionar conocimientos sobre drogas y desmitificar creencias erróneas asociadas a éstas (NIDA, 2003). Este programa ha sido cuidadosa y ampliamente evaluado en los últimos 25 años a través de diversos estudios que han probado su eficacia (Botvin y Griffin, 2005). Las evaluaciones del programa han demostrado consistentemente reducciones de consumo de tabaco, alcohol y marihuana (del 50\% o más) en estudiantes que recibieron el programa en relación con los controles, así como reducciones significativas en el uso de drogas ilegales y mejoras en 
una variedad de factores de riesgo y protectores para el abuso de drogas en adolescentes (Botvin y Griffin, 2007).

En México, existen diversos programas dirigidos a combatir el consumo de drogas en diferentes niveles de intervención (universal, selectiva o indicada). El programa Chimalli es un modelo de prevención basado en los riesgos psicosociales. Su objetivo principal es hacer educación preventiva y crear una protección en los individuos y en las comunidades. Entre las áreas psicosociales en las que trabaja Chimalli se encuentra la prevención del consumo de drogas en niños y adolescentes a través del fortalecimiento de habilidades de protección entre las que se cuentan habilidades sociales, emocionales y cognitivas (Castro, Llanes y Mille, 2000). El programa "Construye tu Vida sin Adicciones" tiene como principal objetivo la conformación de estilos de vida que permitan desarrollar al máximo el potencial de cada persona y propiciar con ello condiciones que mejoren la calidad de vida de las familias y comunidades, por consiguiente prevenir el desarrollo de adicciones. Está dirigido a adolescentes, jóvenes y adultos y cuenta con material específico para cada población (Campollo, 2003). El programa "Yo quiero yo puedo" tiene por objetivo desarrollar en niños las habilidades para la vida que les permitan manejar adecuadamente las situaciones de riesgo y fortalecer factores protectores como la comunicación y la unidad familiar, aumentando el autoconcepto y la habilidad de manejo de presión social, para prevenir el abuso de drogas. Este programa se instrumenta en escuelas públicas para que éstos repliquen el programa con los estudiantes (Pick y Givaudan, 2007).

En términos de responsabilidad social, se hace fundamental abordar y profundizar en la temática del consumo de SPA en adolescentes, con el fin de propiciar reflexión académica frente a los abordajes tradicionales que se han tenido acerca del fenómeno; asimismo, el promover el enfoque de las habilidades para la vida como una propuesta de intervención, desde una mirada crítica, que no se limite al enfoque asistencialista; por el contrario, se configure como un enfoque transversal a la configuración del sujeto.

La Organización Mundial de la Salud -OMS- inicia la promoción de la formación en Habilidades para la vida en diferentes ambientes educativos sobre la década de los años 90, después del reconocimiento que ha existido de las transformaciones culturales y en los estilos de vida, lo que ha generado que en la mayoría de los casos los niños, niñas y adolescentes no estén completamente preparados para enfrentar esos nuevos desafíos a los que los enfrenta el mundo contemporáneo. Partiendo de esta premisa, la Organización Panamericana de la salud -OPS- (2001) viene proponiendo el trabajo con niñas, niños y adolescentes desde un enfoque de las habilidades para la vida el cual 
facilita un desarrollo saludable de la población, basados en múltiple evidencia que demuestra que la formación en las habilidades para la vida previene problemas psicosociales y de salud. Las habilidades para la vida se relacionan directamente con el concepto de competencia social y son entendidas como el grupo de habilidades que favorecen a las personas para enfrentarse con éxito a las exigencias y situaciones de la cotidianidad (Díaz- Alzate y Mejía Zapata, 2018, p. 207).

Esto se relaciona directamente con el modelo de la promoción de la salud, el cual apunta a mejorar la calidad de vida a partir de intervenir los determinantes de la salud. Generalmente va unido al modelo de la prevención de enfermedades, puesto que busca informar sobre los riesgos de las mismas, fortaleciendo en los actores del contexto la capacidad para promover el bienestar (Kornblit, Camarotti y Di Leo, 2010).

La UNICEF (2002) define las habilidades para la vida como recursos psicosociales e interpersonales que ayudan a la gente a tomar decisiones con conocimiento de causa, comunicarse de forma efectiva y desarrollar unos recursos para manejar y autogestionar una vida saludable y productiva. Las habilidades para la vida pueden aplicarse a acciones dirigidas hacia uno mismo, hacia otras personas, o hacia el entorno local. Su objetivo consiste en favorecer la salud y el bienestar en todos estos niveles (Fundación Jacobs, 2011, p.10).

Díaz-Alzate y Mejía Zapata (2018) resaltan que las habilidades para la vida permiten un mejor vivir y se forjan como factores protectores para la prevención de conductas de riesgo como el consumo temprano de SPA; es indiscutible que la capacidad de empatía y de relacionarse con el otro, puede, de alguna manera, beneficiar la construcción de recursos psíquicos para la resistencia ante situaciones de riesgo, especialmente en la adolescencia, cuando el proceso de identificación es fundante de la proporción subjetiva (p. 204).

Por otra parte, se observa que el enfoque de las capacidades propuesto por Martha Nussbaum, resulta una alternativa para repensar el modelo de desarrollo de habilidades para la vida, en tanto "concibe cada persona como un fin en sí misma y no se pregunta solamente por el bienestar total o medio, sino también por las oportunidades disponibles para cada ser humano" (2012a, p. 38), entre tanto es importante reconocer, no sólo la importancia del desarrollo de habilidades que aporten a un mejor vivir, sino vislumbrar las realidades de los adolescentes y los contextos en los cuales viven, los cuales muchas veces son más influyentes de lo que se piensa, esto con el fin de que 
pueda construirse un modelo acorde a las necesidades y oportunidades que se tienen política, social y económicamente.

En base a esto, Nussbaum establece que este enfoque se basa esencialmente en comprender que el desarrollo de estas capacidades permite que el ser humano tenga una vida digna. Define entonces diez capacidades centrales: vida; salud física; integridad física; sentidos, imaginación y pensamiento; emociones; razón práctica; afiliación; relación con otras especies; y control sobre el propio entorno (político y material) (Nussbaum, 2012a, 2012b), que parte de "la idea de la dignidad humana" (Nussbaum, 2012b, p. 163). La incorporación de este enfoque de las capacidades en el enfoque tradicional de habilidades para la vida, supone que no pueden ser conceptos enseñados teóricamente, más bien, releva su importancia a la presunción de un proceso formativo continuo y transversal en las instituciones educativas, para lo cual, debería ser agregado como principio en el currículo de las mismas, si se tiene en cuenta que éstas constituyen un escenario privilegiado de socialización, lo cual aporta directamente a la configuración de subjetividades.

\section{Formación en habilidades blandas}

La formación de habilidades blandas, para Yturralde (2016), es orientar al desarrollo de aptitudes sociales. Es un repertorio de comportamientos capaces de integrar, movilizar y transferir habilidades, juicios y actitudes que agregan valor para la organización y valor social para la persona, la cual permite relacionarse asertivamente con los demás. Entre las habilidades blandas a considerar se encuentran: escuchar activamente, capacidad para comunicarse, capacidad de liderazgo, capacidad de analizar, de juzgar y de automotivarse; conciencia de valores, trabajo en equipo y la capacidad de adaptación al cambio (p. 2-3).

Estas habilidades constituyen la base de todo buen servicio y realización personal, contribuyen a la formación del ser humano y, por lo tanto, son consideradas importantes para la prevención de adicciones en adolescentes. Estas destrezas transversales permiten tener capacidad crítica, emitir pensamientos coherentes basados en valores y principios. Por lo tanto, Suárez (2014) considera que ser habilidoso socialmente implica ser capaz de ponerse en el lugar de la otra persona pero, debe ser capaz de motivar para obtener el máximo de productividad logrando un ambiente agradable de trabajo.

Tanto las habilidades para la vida, como las blandas representan una gran importancia en tanto factores de protección que permiten no solo evitar que el adolescente inicie el consumo de cualquier tipo de sustancia, o desarrolle 
comportamientos adictivos, sino también contribuyen de forma significativa a la calidad de vida de las personas.

\section{Fomento del pensamiento crítico reflexivo}

Se prevé que el desarrollo de habilidades de pensador crítico implica una educación integral, que desarrolle sus competencias y su característica fundamental de ser en el sentido de saber movilizar los conocimientos que se poseen en las diferentes y cambiantes situaciones que se presentan en la práctica (Aznar, Cáceres e Hinojo, 2011).

La capacidad del pensamiento crítico-reflexivo permite analizar con objetividad experiencias e información y ser capaz de llegar a conclusiones propias sobre la realidad. La persona crítica se pregunta, investiga y no acepta las cosas en forma crédula. En términos muy amplios, pensar críticamente significa "pensar con un propósito": demostrar una idea o hipótesis, interpretar el significado de algo o solucionar un problema (Prevención del consumo problemático de drogas, 2012).

El pensamiento crítico depende de dos factores principales: una serie de habilidades cognitivas y las disposiciones afectivas. Lo cognitivo implica aplicar destrezas básicas del pensamiento, como la interpretación, el análisis, la evaluación, la inferencia, la explicación y la autorregulación, en un proceso activo de pensamiento que permite llegar a conclusiones de un orden superior. Las disposiciones afectivas se refieren a la actitud personal; hace falta también querer pensar (Vélez, 2013).

La formación de esta habilidad de pensamiento crítico-reflexivo es una responsabilidad directa de sistemas educativos a nivel internacional, y es una exigencia de la sociedad contemporánea, donde los sistemas educativos ya deben pasar de la formación de sujetos obedientes que se forman para un ciego cumplimiento de órdenes, a formar sujetos pensantes y creativos, que cuestionan el orden existente de cosas, generando soluciones novedosas y alternativas frente a los problemas. Esta habilidad, además de contribuir a avance y progreso social, permite a las personas tomar mejores decisiones a nivel personal y fortalecer el control sobre sus vidas, evitando de esta forma caer en comportamiento adictivos.

\section{Prevención a nivel familiar}

La familia es importante para los adolescentes, ya que su ajuste social y emocional es mejor cuando ésta es cohesiva, expresiva, organizada y fomenta la independencia de sus miembros. De manera inversa, los jóvenes 
tienden a desajustarse cuando perciben que en su familia hay muchos conflictos, demasiado control y una percepción negativa por parte de los padres hacia los hijos. Además, una comunicación eficaz en la familia es un determinante crucial para su bienestar. De la misma manera, es menos probable que los adolescentes que experimentan afecto, cercanía y un desarrollo adecuado de normas en la familia sean influenciados por su grupo de amistades y se involucren en problemas de conducta tales como el consumo de drogas (Martínez, Gómez y Ortega, 2005).

En base a esto, Tellez et al. (2003) afirman que:

La familia puede favorecer o desestimular el consumo de drogas. Una familia permisiva y sin normas claras puede convertirse en un factor que propicia el consumo. La familia que está cerca y cumple una función de regular a sus miembros en el cumplimiento de normas, aparece como factor protector para el consumo de sustancias psicoactivas (p.15).

Existen muchas circunstancias y contextos relacionados con los cambios sociales, estructurales e ideológicos que repercuten en las familias, creando a veces condiciones de riesgo frente al consumo y, otras veces, manteniendo comportamientos que se constituyen en factores protectores (Medina y Ferriani, 2010).

Es necesario tener en cuenta que los factores protectores son antagónicos de los factores de riesgo, sin embargo, la relación no es lineal ni causal, los mismos factores protectores en un individuo pueden ser condiciones de vulnerabilidad para otro, por esta razón es necesario tomar en cuenta tanto el sujeto, como el contexto y la droga.

Para ejemplificar algunas situaciones protectoras, Callisaya Argani (2018) enumera las siguientes: familias "comunicantes", horizontales y con una clara idea de la disciplina, sentido del humor, pasión por asuntos, temas y áreas alternativas al consumo de drogas; infancia "normal", con padres/madres nutricios y aceptantes; comunidad con lazos estrechos y organización clara frente a sus problemas; sentido de trascendencia (incluye, aunque no necesariamente religiosidad o espiritualidad); escuelas afectivas, con profesores afectivos y formadores de habilidades para la vida; control de la delincuencia, incluyendo aquella que involucra la comercialización ilegal de sustancias.

Aunque los factores protectores ante el consumo/abuso de drogas son frecuentemente menos reconocidos, la familia y las habilidades personales según los y las jóvenes tienen gran influencia a la hora de enfrentarse a las drogas (Callisaya Argani, 2018). 
La compenetración familiar constituye el cimiento de la relación entre los padres y los hijos. Estos lazos se pueden fortalecer a través de una capacitación para mejorar la capacidad de los padres para apoyar a sus hijos, la comunicación entre padres e hijos, y la participación de los padres con los hijos (Spoth, Redmond, Trudeau y Shin, 2002; Spoth, Redmond, Shin y Azevedo, 2004).

La comunicación es uno de los factores clave en el clima y cohesión familiar. Tal como lo afirma Cevallos (2008), la comunicación como acción cotidiana posibilita las relaciones sociales y el reconocimiento mutuo. Los seres humanos, como seres en busca de sentido, requieren de la comunicación para encontrarse y establecer relaciones, porque la comunicación es un proceso constructor de sentido colectivo. La comunicación fluida y permanente entre padres e hijos posibilita un mayor involucramiento por parte de los integrantes de la estructura familiar y consolida el núcleo familiar como una red de apoyo para el joven. Con otras palabras, la comunicación es un posibilitador de los factores protectores familiares (Acevedo-Silva, Camargo-Cardona y Fajardo-Ramos, 2016).

Del mismo modo, el involucramiento familiar y la magnitud de apoyo, la calidez familiar, la disciplina y la comunicación familiar son factores relacionados con las interacciones humanas que se gestan en el interior de las familiar como principal escenario de formación de niños y adolescentes. La familia es el factor protector por excelencia, y por lo tanto, el espacio hacia donde se deben encaminar los procesos de prevención al consumo de SPA (Acevedo-Silva, Camargo-Cardona y Fajardo-Ramos, 2016).

Así mismo, los programas de prevención escolar, que suelen incluir a la familia como escenario para la educación contra el consumo de drogas, hacen énfasis en la tarea de informar a los padres sobre los efectos de las sustancias psicoactivas. Sin embargo, dichos programas deberían enfocarse, entre otras cosas, en el mejoramiento de los niveles de comunicación en la familia y propiciar el acompañamiento en tareas escolares, la detección oportuna de dificultades emocionales o académicas y el ejercicio de una autoridad democrática en el hogar.

El monitoreo y la supervisión de los padres son esenciales para prevenir el abuso de las drogas. Se pueden mejorar estas habilidades capacitando a los padres para que aprendan cómo establecer reglas, técnicas para el monitoreo de las actividades de sus hijos, cómo elogiar a los hijos por conducta apropiada, y el uso de disciplina moderada y consistente para hacer respetar las reglas de la familia (Kosterman et al., 2001). 
La educación y la información sobre las drogas para los padres o los cuidadores refuerzan lo que los niños están aprendiendo sobre los efectos dañinos de las drogas y brindan la oportunidad para la discusión familiar sobre el abuso de sustancias legales e ilegales (Bauman et al., 2001).

Las intervenciones cortas enfocadas en la familia y dirigidas a la población en general pueden cambiar de manera positiva las conductas específicas de los padres, las que, a su vez, pueden reducir el riesgo futuro para el abuso de drogas de los hijos (Spoth et al., 2002; Spoth et al., 2004).

En base a lo anterior se puede mostrar como ejemplo dentro de los diferentes marcos de intervención la perspectiva del Positive Youth Development (Benson et al., 2006) (Modelo de Desarrollo Positivo del adolescente), donde la prioridad de la intervención es el fortalecimiento de los factores individuales de protección, contando siempre con la participación del menor y de la familia en todo el proceso. Se trata de una intervención de corte psicoeducativa, poniendo el foco en los recursos personales y sociales y atendiendo siempre al momento en el proceso de crecimiento personal del adolescente. El concepto de desarrollo positivo de adolescente fue inicialmente propuesto por el Search Institute (Scales y Leffert, 1999), y se orienta al fomento de los recursos tanto personales, como familiares y escolares (comunitarios), que permiten crear un sistema de apoyo y las condiciones necesarias para la promoción del desarrollo positivo en los adolescentes.

En consecuencia, la Unidad de Drogas y Adicciones del IPBS de Córdoba (2015), expone los siguientes principios que deben ser los prioritarios y fundamentales en cualquier tipo de intervención asistencial o de corte preventivo con menores y adolescentes que cuenten con un núcleo familiar establecido: reducción de los factores de riesgo y potenciación de los de protección; aprovechamiento de las potencialidades personales y los recursos familiares y sociales con los que cuenta el joven para solucionar sus problemas; potencialización de la intervención individualizada, atendiendo a las características individuales y sociales de cada menor y adolescente así como a su entorno familiar; intervención flexible y dinámica, adaptada al proceso que vaya realizando el menor y su familia; adaptación de los objetivos y metodologías según la etapa evolutiva de la adolescencia; evitación de etiquetas y trato respetuoso; globalidad de la intervención considerando el estilo de vida y el entorno; acompañamiento del adolescente y la familia con el fin de convertir a la familia en el agente principal de mejora en su calidad de vida; coordinación y trabajo, implicando múltiples agentes que están interviniendo con el menor (p. 5-6). 
Por lo tanto, en procesos de intervención familiar para prevenir adicciones, la familia cumple varias funciones importantes en el desarrollo de sus miembros, como afirman Valenzuela, Ibarra, Zubarew y Lorreto (2013), ya que proporciona factores protectores que disminuyen significativamente las conductas de riesgo en el adolescente mediante la instauración de patrones de conductas, pautas y normas de convivencia, creación de un adecuado vínculo entre sus miembros, y un funcionamiento familiar, que cuenta con la disponibilidad de tiempo por parte de los padres para atender las necesidades de sus hijos, comunicación abierta y asertiva y rituales familiares, que fomentan la cohesión y las actividades compartidas.

\section{Prevención a nivel comunitario}

Las estrategias para motivar a una comunidad a actuar dependen de su nivel de preparación, de manera que sus acciones proporcionen los máximos beneficios. Una estrategia para lograr el cambio es a través del establecimiento de alianzas comunitarias anti-drogas.

Estas alianzas implican la realización de reuniones con toda la comunidad, desarrollar campañas de información al público y convocar a patrocinadores interesados en la prevención del consumo de drogas. A fin de fortalecer el impacto de estas estrategias, las alianzas deben concentrarse en la aplicación de programas y enfoques probados científicamente. Se ha demostrado que los medios de comunicación pueden promover la conciencia pública sobre el problema de drogas en la comunidad y prevenir su consumo en poblaciones específicas (Conadic, 2017).

Cabe mencionar que, además de la red familiar y de amigos, la comunidad ejerce control social sobre nuestro comportamiento lo cual puede generar una mayor involucración bajo ciertas condiciones, entre ellas el de contar con una organización comunitaria estructurada y basada en relaciones de cooperación. Uno de los resultados de esta involucración es la capacidad de generar eficacia colectiva y organización social protectora sobre diversos problemas como las del consumo de drogas y la delincuencia (Sampson, Raudenbush y Earls, 1997)

Del mismo modo se presenta la conducta prosocial, la cual se asocia con el establecimiento de conductas de carácter voluntario y empáticas dirigidas al beneficio de otros (Eisenberg, Fabes y Spinrad, 2006), tales como ayudar en los deberes o escuchar a otros cuando quieren hablar sobre un problema.

Según Cabanillas-Rojas (2012) la comunidad barrial, según sus características, puede ser escenario de protección o de riesgo. Gran parte de la inves- 
tigación disponible hace hincapié en la desorganización social, poniéndose en relieve que las características de desventaja o inestabilidad en los barrios están asociadas con problemas de comportamiento individual a través de mecanismos de recursos sociales, control social, socialización colectiva, modelado y estrés. La desorganización social impide la gestión comunitaria para la solución de diversos problemas, que influyen en las personas y las familias. Formulaciones de este tipo apoyan la idea de que la influencia del entorno social puede ser tan relevante como los factores individuales, ofreciendo luces sobre la necesidad de diseñar políticas que articulen estrategias de prevención de consumo a nivel de comunidades.

En este orden de ideas, la comunidad escolar es un espacio por excelencia propicio que puede permitir un desplegué de muchas estrategias preventivas tanto para el consumo de drogas, como en relación a las adiciones comportamentales. Existes diversas propuestas de prevención a nivel universal que se enfocan en los ejes como resiliencia, habilidades para la vida, estilos de vida saludables, ambientes protectores, proyecto de vida (Barceinas, Landeros y Martínez, 2010); prevención de acoso escolar como uno de los factores asociados al consumo (Centros de integración juvenil, 2014); entrenamiento en asertividad, comunicación, atontamiento (Tobler et. al., 2000), los cuáles, a su vez, están orientadas a toda la comunidad educativa, incluyendo estudiantes, padres de familia y docentes. En cuanto a la eficiencia de la prevención en el ámbito escolar, existen tanto estudios que aseveran un efecto positivo de los programas preventivos escolares no solo en prevención de consumo de sustancias, sino en el mejoramiento de habilidades para toma de decisiones y autoestima en los estudiantes (Faggiano et al., 2008), como los que plantean que sus efectos son pequeños y se disminuyen con el tiempo (Canning, Millward, Raj y Warm, 2004). Se ha identificado que los programas de prevención centradas de forma exclusiva en el componente informativo sobre las drogas y sus consecuencias negativas tienen el menor efecto en la población (Gázquez, García del Castillo y Espada, 2009). Además, los programas interactivos que implican la interacción y participación activa de todos los participantes demuestran ser mucho más efectivos (Gázquez, García del Castillo y Espada, 2009).

\section{Prevención de adicciones comportamentales}

Siendo las fuentes bibliográficas relacionadas con las adiciones comportamentales más escasas, el tipo de comportamiento adictivo de mayor abordaje es de adicción al juego y nuevas tecnologías, incluyendo internet, celular, videojuegos y redes sociales. Lo anterior puede explicarse por el he- 
cho de que, aparte de los juegos de azar, ningún otro comportamiento (sexo, trabajo, ejercicio, compras, comida, etc.) aún no se ha clasificado como una verdadera adicción de forma oficial (incluido en el DSM). En una de las revisiones más completas sobre adicciones químicas y conductuales, Sussman, Lisha y Griffiths (2011) examinaron la literatura de prevalencia relacionada con 11 diferentes potencialmente adictivos comportamientos, informando tasas generales de prevalencia de adicciones a fumar cigarrillos (15\%), beber alcohol (10\%), tomar drogas ilícitas(5\%), comer (2\%), juegos de azar (2\%), uso de Internet (2\%), amor (3\%), sexo(3\%), ejercicio (3\%), trabajo (10\%) y compras $(6 \%)$.

Hay muchos factores individuales (vulnerabilidad personal) que pueden participar en la adquisición, desarrollo y mantenimiento de adicciones conductuales (p. ej., rasgos de personalidad, predisposiciones biológicas y genéticas, motivaciones inconscientes, aprendizaje y efectos de acondicionamiento, pensamientos, creencias y actitudes), aunque algunos de los factores son más personales (por ejemplo, la motivación financiera) y presiones económicas en el caso del adicción al juego). Sin embargo, también hay algunos factores de riesgos clave que están altamente asociados con el desarrollo de casi cualquier adicción (química o conductual), como tener antecedentes familiares de adicción, tener comorbilidades de problemas psicológicos, y la falta de supervisión y participación familiar. Factores psicosociales como baja autoestima, la soledad, la depresión, la ansiedad y el estrés parecen ser comunes entre persona con adicciones conductuales (Griffiths, 2015).

Las adiciones comportamentales son más difíciles de identificar a tiempo. Por ejemplo, en casa la adición a las nuevas tecnologías existe una línea delgada entre el uso normal de esta y la adición, considerando que la misma cultura de consumo, el márqueting de cada vez más grande cantidad de productos relacionados con estos, y exponencialmente creciente aceptación de participación y popularidad en los redes sociales como parte de la identidad adolescente, llevan al hecho de que sea difícil identificar los problemas de adicción a tiempo (Arias, Gallego, Rodríguez y del Pozo López, 2012).

El juego patológico es otra de las problemáticas en el ámbito de adiciones comportamentales que afecta a la edad adolescentes nivel mundial (García, Montes y Alcol, 2012), tanto si hablamos de juegos electrónicos como de juegos de azar. Esta adicción, igual como otros comportamientos adictivos, se denomina a veces como la "adicción invisible", ya que los juegos, al igual como en el caso anterior de los aparatos electrónicos, están disponibles en todos los lados y su oferta no solo es aceptada socialmente sino está ampliamente fomentada por los intereses económicos de la filosofía de consumo 
imperante en la sociedad contemporánea. Por esta razón las personas a menudo no se percatan del problema hasta que esta ya llega a manifestaciones extremas.

Lo anterior requiere de una especial atención al respecto de estrategias preventivas, entre los cuales comúnmente se encuentran: proporcionar información sobre el problema de adiciones comportamentales, permitiendo comprender su sintomatología y síntomas de alarma (se realiza tanto con los adolescentes, como con la familia); sensibilización que permite el cambio de actitudes frente a ciertos comportamientos que parecían normales con anterioridad (igualmente se debe trabajar tanto con los adolescentes como con las familias); y formación de hábitos de conducta y/o entrenamiento en estrategias conductuales, donde en el énfasis se hace las en la población adolescente con el fin de prevenir la aparición de adiciones y fomentar un comportamiento sano (Chóliz Montañés, 2017).

En cuanto a la prevención de adiciones comportamentales en adolescentes, la familia y el ambiro escolar son muy importantes. Zeleeva y Shubnikova (2016) plantean que uno de los principales objetivos en la prevención pedagógica es la formación de los recursos individuales resilientes que mejoran la estabilidad de niños y jóvenes frente a las influencias negativas de una sociedad. Hay diferentes clasificaciones de los recursos individuales, por ejemplo, Sirota, Yaltonsky y Viderman (2001) incluyen en este grupo: 1) el nivel de inteligencia (la capacidad y la oportunidad de llevar a cabo una evaluación de problema); 2) formación de un autoconcepto positivo 3) locus de control interno (la capacidad de controlar la vida en general y comportamiento, asumir la responsabilidad por sí mismo); 4) competencia social (la capacidad de comunicarse con los demás y el conocimiento de la realidad social); 5) empatía (la capacidad de empatizar con los demás en el proceso de comunicación); 6) afiliación (el deseo de comunicarse con personas); 7) la actitud de la persona hacia la vida, la muerte, el amor y fe; 8) espiritualidad; 9) estructura motivacional firme y positiva.

La formación de estos recursos personales resilientes tiene que realizarse tanto en el ámbito escolar como familiar. Con este fin es importante realizar talleres de concientización y capacitación a nivel familiar en cuanto a los estilos parentales de autoridad, acompañamiento, uso productivo del tiempo libre, rutinas familiares, entre otros. En el ámbito escolar, a su vez, es de gran importancia fomentar la creación de compañías a nivel global de las comunidades educativas de las instituciones, tal como se está realizando, por ejemplo, con el problema de acoso escolar. Estos programas, siendo transversales e integradas al currículo, deben al igual como en caso de adiciones químicas, 
orientarse a la prevención de adicciones comportamentales. En este orden de ideas, el principal propósito de la escuela hoy en día no es solo trasmitir conocimiento, sino fortalecer a los adolescentes al respecto del manejo de habilidades para la vida y su capacidad resiliente con el fin de prevenir la aparición e instauración de los comportamiento adictivos.

\section{A manera de conclusiones}

Las adicciones son comportamientos que van en contra del ser humano, afectándolo no solo en su parte personal sino en la relación que tiene consigo mismo, con su entorno y con las personas que le rodean ocasionando afectaciones negativas, de allí radica la importancia de su prevención.

La prevención entendida como "toda medida o actuación que tiende a reducir, retrasar o evitar el consumo de determinada sustancia y los problemas derivados de su uso" (Cruz, 2013, p. 25), debe efectuarse desde el personal, familiar o comunitaria, siendo una alternativa viable para mantener alejado al adolescente de adicciones que solo causan daños irreparables en su comportamiento y convivencia.

Es importante resaltar que se le debe seguir apostando a las estrategias preventivas duraderas y estables en el tiempo, para que calen en los adolescentes y jóvenes con el fin de prevenir que estos tomen decisiones erróneas como las adicciones y arruinen su futuro.

La familia se presenta como el grupo de interacción relacionado directamente con el adolescente, el cual debe crear un ambiente de condiciones de desarrollo basadas en la libertad, la justicia, el respeto, la tolerancia, la solidaridad y el diálogo, lo cual favorece la salud mental y genera posibilidades positivas de desarrollo de niños, niñas y adolescentes, contribuyendo a generar factores protectores que les permita hacer frente a los estilos de vida que se imponen en su contexto como la cultura de consumo y facilismo que lleva a generar comportamiento adictivos.

La prevención y el fomento de estrategias en contra de conductas adictivas es un trabajo articulado que comienza desde la familia y que debe ser reforzado por la escuela y la sociedad, partiendo de las premisas de la educación para la vida y la incorporación de actitudes proactivas. 


\section{Referencias}

Acevedo-Silva, M., Camargo-Cardona, L., Fajardo-Ramos, E. (2016). Factores protectores familiares para la prevención del uso de sustancias psicoactivas (SPA) en estudiantes de quinto de primaria de la Institución Educativa San Luis Gonzaga del corregimiento de Chicoral (El Espinal, Tolima). Salud Uninorte, 32 (3), 461-471, http://dx.doi.org/10.14482/sun.30.1.4309

Agencia Nacional de Defensa Jurídica Del Estado (2016). Programa de prevención de consumo de sustancias psicoactivas. Disponible en http://calidad.defensajuridica. gov.co/archivos/GH-PG-05/GH-PG-05V0ProgramaDePrevencionDeSustanciasPsi coactivas.pdf

Albein-Uriosa, N., Martínez-González, J. Gutiérrez, B., Lozano, Ó., Verdejo-García, A. (2011). Bases neurobiológicas de la comorbilidad entre consumo de cocaína y trastornos de la personalidad. Trastornos Adictivos, 13 (1):15-19, Disponible en http://www.elsevier.es/es-revista-trastornos-adictivos-182-articulo-basesneurobiologicas-comorbilidad-entre-consumo-X157509731299626X

Alfonso, J., Huedo-Medina, T., Espada, J. (2009). Factores de riesgo predictores del patrón de consumo de drogas durante la adolescencia. Anales de psicología, 25(2): 330-338, Disponible en http://www.um.es/analesps/v25/v25_2/15-25_2.pdf

Arco, J., Fernández, A. (2002). Porque los programas de prevención no previenen.

International Journal of Clinical and Health Psychology, 2 (2):209-226, Disponible en http://www.redalyc.org/pdf/337/33720201.pdf

Arias, O., Gallego, V., Rodríguez, M., del Pozo López, M. (2012). Adicción a las nuevas

Tecnologías. Psicología de las Adicciones, 1: 2-6, Disponible en https://www.unioviedo. es/gca/uploads/pdf/Psicologia\%20de\%20las\%20Adicciones,\%20vol.1,\%202012. pdf

Ayesta, F. (2002). Bases bioquímicas y neurobiológicas de la adicción al alcohol.

Adicciones, 14(5): 63-78, doi:http://dx.doi.org/10.20882/adicciones.520

Aznar, I., Cáceres, M., Hinojo, M. (2011). La adquisición de competencias específicas en la Educación Superior. Evaluando la formación del psicopedagogo en la Universidad de Granada. Ensayos, 26:71-93. Disponible en https://dialnet. unirioja.es/descarga/articulo/4010568.pdf.

Barceinas, A., Landeros, L., Martínez, G. (2010). Programa Nacional Escuela Segura. 
México:SecretariadeEducaciónPública.Disponibleenhttps://hivhealthclearinghouse. unesco.org/sites/default/files/resources/21436_guiadoce ntes456.pdf

Bauman, K., Foshee, V., Ennett, S., Pemberton, M., Hicks, K., King, T., y Och, G. (2001).

The influence of a family program on adolescent tobacco and alcohol. Am J Public Health, 91(4):604-610. Disponible en: https://ajph.aphapublications.org/doi/ pdf/10.2105/AJPH.91.4.604

Becoña, E. (2006). Adicción a nuevas tecnologías. Vigo: Nova Galícia Edicións.

Becoña, E. (2007). Resiliencia y consumo de drogas: una revisión. Adicciones, 19(1): 89-101, Disponible en http://www.redalyc.org/pdf/2891/289122034010. pdf

Becoña, E. (1999). Bases teóricas que sustentan los programas de prevención de drogas. Plan Nacional Sobre Drogas, 1-309. Disponible en http://www.pnsd.msc. es/Categoria2/publica/pdf/Bases_cientificas.pdf

Benson, P., Scales, P., Hamilton, S., Sesma, A. (with Hong, K., Roehlkepartain, E.). (2006). Positive youth development so far: Core hypotheses and their implications for policy and practice. Search Institute Insights \& Evidence, 3 (1) 1-13, www. searchinstitute.org/research/Insights.

Botvin, G., Griffin, K. (2005). Prevention science, drug abuse prevention, and life skills training: Comments on the state of the science. Journal of Experimental Criminology, 1: 63-78, https://doi.org/10.1007/s11292-004-6462-y

Botvin, G. y Griffin, K. (2007). School-based programmes to prevent alcohol, tobacco and other drug use. International Review of Psychiatry, 19: 607-615. doi:10.1080/09540260701797753.

Cabanillas-Rojas, W. (2012). Factores de riesgo/protección y los programas preventivos en drogodependencias en el Perú. Revista Peruana de Medicina Experimental y Salud Publica, 29(1): 104-111. Disponible en http://www.scielo. org.pe/scielo.php? script $=$ sci_arttext\&pid $=$ S1726-46342012000100016\&lng $=$ es \&tlng $=$ es.

Cáceres, D., Salazar, I., Varela, M., Tovar, J. (2006). Consumo de drogas en jóvenes universitarios y su relación de riesgo y protección con los factores psicosociales. Universitas Psychologica, 5(3): 521-534. Disponible en http://www.redalyc.org/ pdf/647/64750308.pdf

Castro, M., Llanes, J., Mille C. (edit) (2000). Construye tu vida sin adicciones. Guía para el facilitador. México: CONADIC. 
Callisaya Argani, J. I. (2018). Factores de riesgo y protección ante el consumo de drogas en jóvenes del municipio de La Paz. Drugs and Addictive Behavior, 3(1): 52-74. Doi: https://doi.org/10.21501/24631779.2635

Camarotti, A., Kornblit, A. (2015). Abordaje integral comunitario de los consumos problemáticos de drogas: construyendo un modelo. Salud colectiva, 11(2):211221, Disponible en http://www.scielo.org.ar/pdf/sc/v11n2/v11n2a05.pdf

Campollo R, O. (2003). Modelos de Prevención. Centro de Estudios de Alcoholismo y Adicciones. CUCS. Universidad de Guadalajara, Disponible en http://www.cucs. udg.mx/investigacion/calcohol/principal.htm

Canning, U., Millward, L., Raj, T. y Warm, D. (2004). Drug use prevention among young people: a review of reviews. London: Health Development Agency. http:// www.emcdda.europa.eu/attachements.cfm/att_93977_EN_Health\%20Developm ent\%20Agency\%20Review\%20of\%20Reviews.pdf

Centros de integración juvenil (2014). Acoso escolar y consumo de drogas: Estrategias para su prevención. México: Secretaria de educación básica. Disponible en http:// www.intranet.cij.gob.mx/Archivos/Pdf/PrevBulling/MetodologiaPrevencionBull ying.pdf

Cid-Monckton, P., Pedrao, L. (2011). Factores familiares protectores y de riesgo relacionados al consumo de drogas en adolescentes. Rev. Latino-Am. Enfermagem, 19:738-745, http://dx.doi.org/10.1590/S0104-11692011000700011

Chóliz Montañés, M. (2017). Prevención de las adicciones tecnológicas en la adolescencia. Journal of Parents and Teachers, 369: 53-59, doi:https://doi. org/10.14422/pym.i369.y2017.008.

Conadic (2017). Movilización comunitaria en la prevención de las adicciones. En. Tavera, S., Martínez, M. (edit). Prevención de las adicciones y promoción de conductas saludables para una nueva vida. México: Sistema Nacional para el Desarrollo Integral de la Familia, Cap. V: pp. 65-108, Disponible en: http://www. conadic.salud.gob.mx/pdfs/nueva_vida/prevad_cap6.pdf

Cruz, F. (2013). Uso de las nuevas tecnologías en los programas de prevención. Adaptación del programa "Entre Todos". Recuperada de http://dspace.unia.es/ bitstream/handle/10334/2838/0577_Cruz. pdf?sequence $=1$

De la Villa Moral, M., Ovejero, A. (2005). Modificación de las actitudes, hábitos y frecuencia de consumo de alcohol y otras sustancias psicoactivas en adolescentes españoles a partir de un programa educativo-preventivo. Revista Colombiana de Psicología, 14: 100-118, http://www.redalyc.org/pdf/804/80401410.pdf 
De la Villa Moral, M., Rodríguez, F., Ovejero, A. (2010). Correlatos psicosociales del consumo de sustancias psicoactivas en adolescentes españoles. Salud Pública de México, 52(5), 406-415. Recuperado en 24 de noviembre de 2017, de http://www.scielo.org.mx/scielo.php?script=sci_arttext\&pid=S0036$36342010000500008 \& \ln g=$ es\&tlng $=$ es.

Díaz-Alzate, M., Mejía Zapata, S. (2018). Desarrollo de habilidades para la vida en la prevención del consumo de sustancias psicoactivas: un enfoque crítico al modelo existente. El Ágora, 18(1), 203-210. doi:http:// dx.doi. org/10.21500/16578031.3450

Eisenberg, N., Fabes, R., Spinrad, T. (2006). Prosocial development. En W. Damon y N.

Eisenberg (eds.), Handbook of child psychology, vol. 3: Social, emotional and personality development (pp. 646-718). Nueva York: John Wiley \& Sons

Faggiano, F., Vigna-Taglianti, F. D., Versino, E., Zambon, A., Borraccino, A., Lemma, P. (2008). School-based prevention for ilicit drug use. A systematic review. Preventive Medicine, 46, 385-396. DOI: 10.1016/j.ypmed.2007.11.012

Fundación Jacobs (2011). Seguimiento y Evaluación de las Habilidades para la vida orientadas al desarrollo de la juventud. Suiza: Layout Prepress Printing. Disponible en http://www.habilidadesparaadolescentes.com/archivos/Evaluacion_de_ habilidades_par a_la_vida.pdf

García, A., Montes, C., Alcol, I. (2012). El juego patológico en los adolescentes. Psicología de las Adicciones, 1: 29-34, https://www.unioviedo.es/gca/uploads/ pdf/Psicologia\%20de\%20las\%20Adicciones,\%20vol.1,\%202012.pdf

García del Castillo, J. (2013). Adicciones tecnológicas: el auge de las redes sociales. Salud y drogas, 13(1): 5-13, http://www.redalyc.org/pdf/839/83928046001.pdf

Gázquez, M., García del Castillo, J., Espada, J. (2009). Características de los programas eficaces para la prevención escolar del consumo de drogas. Salud y drogas, 9(2): 185-208, http://www.redalyc.org/pdf/839/83912988004.pdf

Gómez Cobos, E. (2008). Adolescencia y familia: revisión de la relación y la comunicación como factores de riesgo o protección, Revista Intercontinental de Psicología y Educación, 10 (2): 105-122. Disponible en: http://www.redalyc.org/ pdf/802/80212387006.pdf

Gómez Rodríguez, D., Gutiérrez Herrera, M., Londoño, S. (2013). Depresión y consumo de sustancias psicoactivas en adolescentes en la ciudad de Bogotá. Psychologia, Avances de la disciplina, 7 (1), pp. 45-51. Disponible en: http:// www.scielo.org.co/pdf/psych/v7n1/v7n1a05.pdf 
Gonzálvez, M., Espada, J., Guillén-Riquelme, A., Secades, R., Orgilés, M. (2016). Asociación entre rasgos de personalidad y consumo de sustancias en adolescentes españoles. Adicciones, 28(2): 108-115, Disponible en http://www.adicciones.es/ index.php/adicciones/article/viewFile/777/733

Griffiths, M. (2015). Classification and treatment of behavioural addictions. Clinical Behavioural Addictions, 2: 44-46, https://www.researchgate.net/profile/ Mark_Griffiths2/publication/273948452_Classific ation_and_treatment_of_ behavioural_addictions/links/551123420cf2a8dd79bfcc4c/Cla ssification-andtreatment-of-behavioural-addictions.pdf

Horcajadas, F., Castillo, I., Mangado, E. (2010). Manual de psicólogos especialistas en psicología clínica en formación. Disponible en http://www.fundacioncsz.org/ ArchivosPublicaciones/270.pdf

Jáuregui, I. (2002). Una perspectiva cultural de la adicción. Gazeta de Antropología, 18, art 07, Disponible en http://hdl.handle.net/10481/7394

Jiménez, M., Ovejero Bernal, A. (2003). Actitudes ante el consumo de sustancias psicoactivas y mentalidades del usuario en adolescentes de Secundaria. Entemu, 15:151-175. Disponible en http://info.uned.es/ca-gijon/web/actividades/ publica/entemu03/a6.pdf

Kornblit, A., Camarotti. A., Di Leo, P. (2010). Prevención del consumo problemático de drogas. Modulo I: La construcción social de la problemática de las drogas. UNICEF. Recuperado de http://files.unicef.org/ argentina/spanish/Edu_ModulosESI.pdf

Kosterman, R., Hawkins, J., Haggerty, K., Spoth, R., Redmond, C. (2001). Preparing for the drug free years: Session-specific effects of a universal parent-training intervention with rural families. J Drug Educ 31(1):47-68. Disponible en: http:// journals.sagepub.com/doi/10.2190/3KP9-V42V-V38L-6G0Y

Llano Sierra, L., Martínez Saldarriaga, M. G., Torres Benítez, M., Ochoa Parra, J. M. y Peláez Olarte, D. (2017). Prevención del consumo de sustancias psicoactivas. Revisión teórica de los modelos y estrategias preventivas. Drugs and Addictive Behavior, 2(1),131-141. Doi: http://dx.doi.org/10.21501/24631779.2266

Llorens Aleixandre, N., Perelló del Río, M., Palmer Pol, A. (2004). Estrategias de afrontamiento: factores de protección en el consumo de alcohol, tabaco y cannabis. Adicciones, 16 (4):1-6, http://www.adicciones.es/index.php/adicciones/article/ view/391

López, M., Vilariño, C., Linares, E., González, J. (2014). Consumo de sustancias psicoactivas en una muestra de jóvenes universitarios. Psicología y Salud, 13(1): 5-17. Disponible en http://revistas.uv.mx/index.php/psicysalud/article/ viewFile/934/1723 
López-Torrecillas, F., Peralta, I., Muñoz-Rivas, M. J., Godoy, J. F. (2003). Autocontrol y consumo de drogas. Adicciones, 15(2):127-136. http://www.adicciones.es/ index.php/adicciones/article/view/436

Mangrulkar, L., Whitman, C., Posner, M. (2001). Enfoque de habilidades para la vida para un desarrollo saludable de niños y adolescentes. Washington, DC: Organización Panamericana de la Salud. Disponible en http://www.codajic.org/sites/www. codajic.org/files/Enfoque\%20de\%20Habilidades\%20para\%20la\%20vida\%20 OPS_0.pdf

Mariño, N., Castro, J., Torrado, J. (2012). Funcionamiento ejecutivo en policonsumidores de sustancias psicoactivas. Revista de Psicología Universidad de Antioquia, 4 (2), 49-64, Disponible en https://dialnet.unirioja.es/descarga/ articulo/4865179.pd

Martínez, J., Verdejo, A (2011). Creencias básicas adictivas y craving. Adicciones, 23(1):339-352, http://www.adicciones.es/index.php/adicciones/article/ view/166/166

Martínez, P., Gómez, A., Ortega, S. (2005). Adicciones y Patrones Familiares de Conducta. Psicología Iberoamericana, 13 (1), 5-11. http://www.redalyc.org/ pdf/1339/133926982003.pdf

Medina, N., Ferriani, M. (2010). Factores protectores de las familias para prevenir el consumo de drogas en un municipio de Colombia. Revista Latino-Americana de Enfermagem, 18(spe): 504-512. https://dx.doi.org/10.1590/S010411692010000700004

Melo, D., Castanheira, L. (2010). Autoeficacia y actitud hacia el consumo de drogas en la infancia: explorando los conceptos. Rev. Latino-Am. Enfermagem, 18(Spec):655662, http://www.scielo.br/pdf/rlae/v18nspe/a24v18nspe.pdf

Muñoz Rivas, M., Graña López, J. (2001). Factores familiares de riesgo y de protección para el consumo de drogas en adolescentes. Psicothema, 13 (11):8794. Disponible en http://www.redalyc.org/pdf/727/72713113.pdf

Musayón, Y., Torres, C., Sánchez, E., Chavéz, E. (2005). Factores de riesgo del consumo de bebidas alcohólicas en escolares de educación secundaria. Investigación y Educación en Enfermería, 23(1), 54-67. Disponible en http://www.scielo.org.co/ scielo.php? script $=$ sci_arttext\&pid $=$ S0120-53072005000100005\&lng $=$ en\&tlng $=$ es.

Musitu, G., Jiménez, T., Murgui, S. (2007). Funcionamiento familiar, autoestima y consumo de sustancias en adolescentes: un modelo de mediación. Salud Pública de México, 49(1), 3-10. Disponible en http://www.scielo.org.mx/scielo. php?script $=$ sci_arttext\&pid=S0036-36342007000100002\&lng =es\&tlng=es. 
National Institute of Drug Abuse (NIDA) (2003). Preventing drug use among children and adolescents. A research based guide for parents, educators and community leaders. Second Edition. Maryland: National Institute of Drug Abuse and National Institute of Health. Disponible en https://www.drugabuse.gov/sites/default/ files/preventingdruguse_2.pdf

Nussbaum, M. (2012a). Crear capacidades. Barcelona: Paidós Ibérica.

Nussbaum, M. (2012b). Las fronteras de la justicia. Consideraciones sobre la exclusión. Barcelona: Paidós Ibérica.

ODC (Observatorio de Drogas de Colombia) (2014). Estudio Nacional de consumo de sustancias psicoactivas en Colombia, 2013. Gobierno Nacional de la Republica de Colombia. Disponible en https://www.unodc.org/documents/colombia/2014/ Julio/Estudio_de_Consumo_UNOD C.pdf

Organización Mundial de la Salud (OMS) (1993). Enseñanza en los colegios de las habilidades para vivir. Ginebra: Organización Mundial de la Salud. Disponible en http://centroderecursos.alboan.org/ebooks/0000/0148/Ense\%C3\%B1anza_ en_los_cole gios_de_las_habilidades_para_la_vida.pdf

Organización Panamericana de la Salud (OPS) (2001). Enfoque de habilidades para la vida para un desarrollo saludable de niños y adolescentes. Washington, DC. Disponible en http://www1.paho.org/hq/dmdocuments/2009/enfoquehabilidades-vida-desarrollo- saludable-ninos-adolescentes-asdi-kellog.pdf

Ortiz, J., Lopera, N., González, N., Klimenko, O. (2017). Actitudes de favorabilidad hacia conductas adictivas prevalentes en una muestra de adolescentes entre 12 y 18 años de algunas Instituciones Educativas en Medellín y Ríosucio. Revista Psicoespacios, 11(19): 24-45, https://doi.org/10.25057/issn.2145-2776

Otero-López, J., Villardefranco, E. (2011). Materialismo y adicción a la compra: Examinando el papel mediador de la autoestima. Boletín de Psicología, 103: 45-59, Disponible en http://www.uv.es/seoane/boletin/previos/N103-3.pdf

Páramo, M. (2011). Factores de Riesgo y Factores de Protección en la Adolescencia: Análisis de Contenido a través de Grupos de Discusión. Terapia psicológica, 29(1), 85-95. Disponible en https://dx.doi.org/10.4067/S0718-48082011000100009

Pérez Rave, J. (2012). Revisión sistemática de literatura en ingeniería. Medellín: Universidad de Antioquia.

Pick, S., Givaudan, M. (2007). Yo quiero, yo puedo: Estrategia para el desarrollo de habilidades y competencias en el sistema escolar. Disponible en http://pepsic. bvsalud.org/pdf/psie/n23/v23a11.pdf 
Prevención del consumo problemático de drogas (2012). Pensamiento crítico. Disponible en: http://files.unicef.org/argentina/spanish/Edu_ModulosESI.pdf

Rueda, E., Monreal, M. (2012). Consumo de sustancias en la adolescencia: un análisis de las diferencias desde el punto de vista psicosocial. IV Congreso Universitario Nacional Investigación y Género, Ministerio de Educación y Ciencia de España. Disponible en https://idus.us.es/xmlui/bitstream/handle/11441/40667/Pages\%20from\%20 Investigacio n_Genero_12-1509-2188-5.pdf? sequence $=1$ \&isAllowed $=\mathrm{y}$

Sampson RJ, Raudenbush SW, Earls F. (1997). Neighborhoods and violent crime: a multilevel study of collective efficacy. Science, 277(5328):918-924. Disponible en http://science.sciencemag.org/content/277/5328/918.full

Scales, P., Leffert, N. (1999). Developmental assets: A synthesis of the scientific research on adolescent development. Minneapolis: Search Institute, Disponible en https://issuu.com/search-institute/docs/dassets

Sirota, N., Yaltonsky, V., Viderman, N. (2001). Prevention of addiction in adolescents. Moscow: Genesis.

Slapak, S., Grigoravicius, J. (2007). "Consumo de drogas": la construcción de un problema social. Anuario de Investigaciones, XIV; Disponible en http://www.scielo. org.ar/pdf/anuinv/v14/v14a23.pdf

Spoth, R., Redmond, C., Shin, C., Azevedo, K. (2004). Brief family intervention effects on adolescent substance initiation: School-level growth curve analyses 6 years following baseline. Journal Consult Clin Psychol, 72(3):535-542. Disponible en https://www.ncbi.nlm.nih.gov/pubmed/15279537

Spoth, R., Redmond, C., Trudeau, L., Shin, C. (2002). Longitudinal substance initiation outcomes for a universal preventive intervention combining family and school programs. Psychol Addict Behav 16(2):129-134. Disponible en: https:// www.ncbi.nlm.nih.gov/pubmed/12079251

Suárez, J. (2014). Habilidades blandas: Indispensables para ser un profesional exitoso. Disponible en http://www.rinconpsicologia.com/2014/03/habilidadesblandas- indispensables-para.html\# comment-form

Sussman, S, Lisha, N, Griffiths, M. (2011). Prevalence of the addictions: A problem of the majority or the minority? Eval Health Professions 34(1):3-56, doi: $10.1177 / 0163278710380124$

Téllez, J, Cote M, Savogal F, Martínez E, Cruz U. (2003). Identificación de factores protectores en el uso de sustancias psicoactivas en estudiantes universitarios. Rev Fac Med, 51(1):15-24. Disponible en: http://bases.bireme.br/cgi- bin/wxislind. 
exe/iah/online $/$ ? IsisScript $=$ iah/iah.xis\&src $=$ google\&base $=$ ADOLEC $\&$ lang $=p \&-$ nextAction $=\operatorname{lnk} \&$ exprSearch $=424538$ \&indexSearch $=$ ID

Tobler, N. S., Roona, M. R., Ochshorn, P., Marshall, D. G., Streke, A. V., y Stackpole, K. M. (2000). School-based adolescent drug prevention programs: 1998 meta-analysis. Journal of Primary Prevention, 20(4), 275-336. DOI: https://doi. org/10.1023/A:1021314704811

Unidad de Drogas y Adicciones del IPBS de Córdoba (2015). Programa de intervención con adolescentes y familias. Disponible en: http://www.ipbscordoba.es/uploads/ Documentos/2017/Programa_de_Intervencion_con_menores,_adolescentes, jovenes_y_familias.pdf

UNODC (2010). La prevención en las manos de los y las jóvenes. Disponible en: http://www.odc.gov.co/Portals/1/publicaciones/pdf/consumo/recursos/ CO031052010-prevencion_manos_jovenes.pdf

UNODC (2015). Informe Mundial sobre las drogas. Oficina De Naciones Unidas Contra el Delito y la Droga. Disponible en http://www.unaids.org/es/resources/ presscentre/featurestories/2015/june/20150626_wdr

Valenzuela, M., Ibarra, A., Zubarew, T., Lorreto, M. (2013). Prevención de conductas de riesgo en el Adolescente: rol de familia. Index de Enfermería, 22(1-2): 50-54. https://dx.doi.org/10.4321/S1132-12962013000100011

Vara Horna, A (2010). ¿Cómo evaluar la rigurosidad científica de las tesis doctorales? Criterios teóricos y metodológicos aplicados a la educación. Universidad San Martin de Porras: Fondo editorial, Disponible en https://www.researchgate.net/ publication/215797775_Como_evaluar_la_rigurosidad_c ientifica_de_las_tesis_ doctorales

Vargas, A., Londoño, N. (2016). Creencias asociadas al consumo y dependencia de sustancias psicoactivas. Revista Katharsis, 21: 111-130. Disponible en http:// revistas.iue.edu.co/index.php/katharsis

Vélez, C. (2013). Una reflexión interdisciplinar sobre el pensamiento crítico. Revista Latinoamericana de Estudios Educativos, 2(9): 11-39. http://www.redalyc.org/ html/1341/134135724002/

Villarreal, M. Sánchez, J., Musitu, G. (2013). Análisis psicosocial del consumo de alcohol en adolescentes mexicanos. Universitas Psychologica, 12(3): 857-873, doi:10.11144/Javeriana.UPSY12-3.apca

Wille-González, F. (2011). Bases neurológicas de la dependencia a sustancias psicoactivas desde la mirada social. Revista Costarricense de Psicología, 19(1) 7381. Disponible en http://www.redalyc.org/pdf/4767/476748709001.pdf 
Yturralde, E. (2016). El desarrollo sostenido de las habilidades y competencias. Disponible en http://www.habilidadesblandas.com/

Zeleeva, V., Shubnikova, E. (2016). Prevention of addictive behavior based on the formation of teenagers' resilience. International Journal of environmental \& Science education, 11(8): 2015-2023, DOI: 10.12973/ijese.2016.574a 